\title{
SUCCESSIVE ITERATIONS AND LOGARITHMIC MEANS
}

\author{
ÁdÁm Besenyei, DÉnes Petz
}

Abstract. The successive iteration (started by Lagrange and Gauss) produces a new mean from two given ones. Several examples of matrix means are given that require the proof of the matrix monotonicity of the corresponding representing function. The paper contains extensions of the logarithmic mean and it is obtained that the Stolarsky mean can be used also for matrices.

Mathematics subject classification (2010): Primary 15A42; Secondary 47A64.

Keywords and phrases: Matrix monotone function, matrix mean, Gauss's arithmetic-geometric mean, logarithmic mean, Stolarsky mean.

\section{REFERENCES}

[1] T. ANDo, Concavity of certain maps and positive definite matrices and applications to Hadamard products, Linear Alg. Appl. 26 (1979), 203-241.

[2] Á. BeSEnYei And D. Petz, Completely positive mappings and mean matrices, Linear Algebra Appl. 435 (2011), 984-997.

[3] R. Bhatia, Matrix Analysis, Springer, New York, 1996.

[4] R. Bhatia, The logarithmic mean, Resonance, 13 (2008), 583-594.

[5] R. Bhatia And R-C. Li, An interpolating family of means, Commun. Stoch. Anal., 6 (2012) 15-31.

[6] J. M. BorWeIn AND P. B. BorWeIn, Pi and the AGM: A study in analytic number theory and computational complexity, John Wiley \& Sons Inc., New York, 1987.

[7] D. A. Cox, The arithmetic-geometric mean of Gauss, Enseign. Math. 30 (1984), 275-330.

[8] F. Kubo And T. Ando, Means of positive linear operators, Math. Ann. 246 (1980), 205-224.

[9] E. A. Morozova, N. N. Chentsov, Markov invariant geometry on state manifolds (Translated from Russian), J. Soviet Math., 56 (1991), 2648-2669.

[10] E. Neumann And Zs. PÁLes, On comparison of Stolarsky and Gini means, J. Math. Anal. Appl., 278 (2003), 274-285.

[11] D. PeTZ, Monotone metrics on matrix spaces, Linear Algebra Appl. 244 (1996), 81-96

[12] D. Petz, Covariance and Fisher information in quantum mechanics, J. Phys. A: Math. Gen. 35 (2002), 929-939.

[13] G. M. Phillips, Two Millennia of Mathematics From Archimedes to Gauss, Springer, New York, 2000.

[14] K. B. Stolars ky, Generalizations of the logarithmic mean, Math. Mag. 48 (1975), 87-92. 